\title{
Some classes of homeomorphisms that preserve multiplicity and tangent cones
}

\author{
J. Edson Sampaio \\ Dedicated to Professor Lê Dũng Tráng on the occasion of his 70th birthday \\ ABSTRACT. In this paper it is presented some classes of homeomorphisms that \\ preserve multiplicity and tangent cones of complex analytic sets. Moreover, \\ we present a class of homeomorphisms that has the multiplicity as an invariant \\ when we consider right equivalence and this class contains many known classes \\ of homeomorphisms that preserve tangent cones. Finally, we show versions of \\ these results looking at infinity.
}

\section{Introduction}

In 1971, O. Zariski in [16 propose many questions and the most known among of them is the following

Question A Let $f, g:\left(\mathbb{C}^{n}, 0\right) \rightarrow(\mathbb{C}, 0)$ be two complex analytic functions. If there is a homeomorphism $\varphi:\left(\mathbb{C}^{n}, V(f), 0\right) \rightarrow\left(\mathbb{C}^{n}, V(g), 0\right)$, then is it true that $m(V(f), 0)=m(V(g), 0)$ ?

Another question made in [16] was Question B and it is presented below a weak version of it.

Question B' Let $f, g:\left(\mathbb{C}^{n}, 0\right) \rightarrow(\mathbb{C}, 0)$ be two complex analytic functions. If there is a homeomorphism $\varphi:\left(\mathbb{C}^{n}, V(f), 0\right) \rightarrow\left(\mathbb{C}^{n}, V(g), 0\right)$, then is there a homeomorphism $h: \mathbb{P} C(V(f), 0) \rightarrow \mathbb{P} C(V(g), 0)$ ?

In Question B', $\mathbb{P} C(V(f), 0)$ and $\mathbb{P} C(V(g), 0)$ are, respectively, the projectivezed cones of $C(V(f), 0)$ and $C(V(g), 0)$.

Question A is still an open problem. However, J. F. de Bobadilla in [4 showed that Question B' (and, in particular, Question B) has an negative answer.

Here, we want also consider a weak version of Question A that it is presented above:

2010 Mathematics Subject Classification. Primary MSC 14B05 - MSC 32S50.

Key words and phrases. Multiplicity - Tangent cones - Zariski's Problems.

The author was supported by the ERCEA 615655 NMST Consolidator Grant and also by the Basque Government through the BERC 2014-2017 program and by Spanish Ministry of Economy and Competitiveness MINECO: BCAM Severo Ochoa excellence accreditation SEV-2013-0323. 
Question $\mathbf{A}^{\prime}$ Let $f, g:\left(\mathbb{C}^{n}, 0\right) \rightarrow(\mathbb{C}, 0)$ be two reduced complex analytic functions. If there is a homeomorphism $\varphi:\left(\mathbb{C}^{n}, 0\right) \rightarrow\left(\mathbb{C}^{n}, 0\right)$ such that $f=g \circ \varphi$, then is it true that $m(V(f), 0)=m(V(g), 0)$ ?

Let me remark that by using Corollary in $\mathbf{9}$ together with Corollary 2 in $\mathbf{1 2}$, we obtain that, in the case of functions with isolated singularities, Question A' is equivalent to Question A.

Finally, let me state the following question which is related with Question B':

Question B" Let $f, g:\left(\mathbb{C}^{n}, 0\right) \rightarrow(\mathbb{C}, 0)$ be two complex analytic functions. If there is a homeomorphism $\varphi:\left(\mathbb{C}^{n}, V(f), 0\right) \rightarrow\left(\mathbb{C}^{n}, V(g), 0\right)$, then is there a homeomorphism $h:(C(V(f), 0), 0) \rightarrow(C(V(g), 0), 0)$ ?

Thus we want study some classes of homeomorphisms that satisfy or do not satisfy the Questions A' and B". Moreover, we show in this paper that Questions A', B' and B" are related.

\section{Classes of homeomorphisms that preserves tangent cones}

Let us define the exact definition of tangent cone that it is used in this paper.

Definition 2.1. Let $A \subset \mathbb{R}^{n}$ be a set such that $x_{0} \in \overline{A \backslash\left\{x_{0}\right\}}$. We say that $v \in \mathbb{R}^{n}$ is a tangent vector of $A$ at $x_{0}$ if there is a continuous curve $\alpha:[0, \varepsilon) \rightarrow \bar{A}$ satisfying $\alpha((0, \varepsilon)) \subset A$ and $\alpha_{i}(t)-x_{0}=t v+o(t)$, where $g(t)=o(t)$ means that $\lim _{t \rightarrow 0^{+}} \frac{g(t)}{t}=0$. Let $C\left(A, x_{0}\right)$ be the set of all tangent vectors of $A$ at $x_{0}$. We call $C\left(A, x_{0}\right)$ the tangent cone of $A$ at $x_{0}$.

Definition 2.2. Let $H$ be a set of germs of homeomorphisms between germs of complex analytic sets at the origin of some $\mathbb{C}^{n}$. We say that $H$ is a class of germs of homeomorphisms that preserves tangent cones, if whenever that $h:(X, 0) \rightarrow(Y, 0)$ belongs to $H$, then there exists $\phi:(C(X, 0), 0) \rightarrow(C(Y, 0), 0)$ such that $\phi \in H$.

Definition 2.3. Let $p$ be a point in $\mathbb{R}^{n}$. The mapping $\rho_{n, p}: \mathbb{S}^{n-1} \times \mathbb{R}^{+} \rightarrow \mathbb{R}^{n}$ given by $\rho_{n, p}(x, r)=r x+p$ is called spherical blowing-up (at $\left.p\right)$ of $\mathbb{R}^{n}$.

Definition 2.4. Let $X$ be a subset of $\mathbb{R}^{n}$ and $p \in X$. The strict transform (at $p$ ) of the subset $X$ under the spherical blowing-up $\rho_{n, p}$ is $X_{p}^{\prime}:=\overline{\rho_{n, p}^{-1}(X \backslash\{p\})}$ and the boundary $\partial X_{p}^{\prime}$ of the strict transform (at $\left.p\right)$ is $\partial X_{p}^{\prime}:=X_{p}^{\prime} \cap\left(\mathbb{S}^{n-1} \times\{0\}\right)$.

Remark that when $X$ is a subanalytic subset, we have $\partial X_{p}^{\prime}=\mathbb{S}_{p} X \times\{0\}$, where $\mathbb{S}_{p} X=C(X, p) \cap \mathbb{S}^{n-1}$.

Let $X \subset \mathbb{R}^{n}$ and $Y \subset \mathbb{R}^{m}$ be two subsets. Let us recall some definitions about differentiability of mappings: we say that a mapping $f: X \rightarrow Y$ is a $C^{k}(k \geq 1)$ mapping, if for each $x \in X$, there exist an open $U \subset \mathbb{R}^{n}$ and a mapping $F: U \rightarrow \mathbb{R}^{m}$ such that $x \in U,\left.F\right|_{X \cap U}=\left.f\right|_{X \cap U}$ and $F$ is a $C^{k}$ mapping. We say that a mapping $f: X \rightarrow Y$ is differentiable at $x \in X$, if there exist an open $U \subset \mathbb{R}^{n}$ and a mapping $F: U \rightarrow \mathbb{R}^{m}$ such that $x \in U,\left.F\right|_{X \cap U}=\left.f\right|_{X \cap U}$ and $F$ is differentiable at $x$.

Definition 2.5. We say that a mapping $h:\left(X, x_{0}\right) \rightarrow\left(Y, y_{0}\right)$ is weakly directional differentiable if for each $v \in C\left(X, x_{0}\right)$ and for any two curves $\alpha_{1}, \alpha_{2}$ : $[0, \varepsilon) \rightarrow X$ satisfying $\alpha_{i}(0)=x_{0}$ and $\alpha_{i}(t)-x_{0}=t v+o(t)$ for $i=1,2$, we have that the limit $\lim _{t \rightarrow 0^{+}} \frac{h\left(\alpha_{i}(t)\right)-h\left(y_{0}\right)}{t}$ there exists for $i=1,2$ and these two limits are 
equal. In this case, we have a mapping $D^{+} h_{x_{0}}: C\left(X, x_{0}\right) \rightarrow C\left(Y, y_{0}\right)$ such that for each $v \in C\left(X, x_{0}\right)$, it is given by

$$
D^{+} h_{x_{0}}(v)=\lim _{t \rightarrow 0^{+}} \frac{h(\alpha(t))-h\left(y_{0}\right)}{t},
$$

where $\alpha:[0, \varepsilon) \rightarrow X$ satisfies $\alpha_{i}(0)=x$ and $\alpha(t)-x_{0}=t v+o(t)$.

The above definition is a little bit more general than the definition presented in [15] in the case of subsets of Euclidean spaces.

Definition 2.6. Let $\left(X, x_{0}\right)$ and $\left(Y, y_{0}\right)$ be subsets germs, respectively, at $x_{0} \in \mathbb{R}^{n}$ and $y_{0} \in \mathbb{R}^{m}$. We say that a homeomorphism $h:\left(X, x_{0}\right) \rightarrow\left(Y, y_{0}\right)$ is a:

- $C^{1}$ equivalence (between $\left(X, x_{0}\right)$ and $\left.\left(Y, y_{0}\right)\right)$, if there are representatives of $h$ and $h^{-1}$ such that they are $C^{1}$ mappings. In this case, we say that $\left(X, x_{0}\right)$ and $\left(Y, y_{0}\right)$ are $C^{1}$ equivalent. We denote by $H_{D i f f\left(C^{1}\right)}$ to be the set of all $C^{1}$ equivalences between germs of complex analytic sets at the origin of some complex Euclidean space.

- differentiable equivalence (between $\left(X, x_{0}\right)$ and $\left.\left(Y, y_{0}\right)\right)$, if $h$ and $h^{-1}$ are respectively differentiable at $x_{0}$ and $y_{0}$. In this case, we say that $\left(X, x_{0}\right)$ and $\left(Y, y_{0}\right)$ are differentiable equivalent. We denote by $H_{D i f f}$ to be the set of all differentiable equivalences between germs of complex analytic sets at the origin of some complex Euclidean space.

- weak directional equivalence (between $\left(X, x_{0}\right)$ and $\left.\left(Y, y_{0}\right)\right)$, if $h$ and $h^{-1}$ are respectively weakly directional differentiable and

$$
D^{+} h_{x_{0}}: C\left(X, x_{0}\right) \rightarrow C\left(Y, y_{0}\right)
$$

is a homeomorphism. In this case, we say that $\left(X, x_{0}\right)$ and $\left(Y, y_{0}\right)$ are weak directional equivalent. We denote by $H_{w D i r}$ to be the set of all weak directional equivalences between germs of complex analytic sets at the origin of some complex Euclidean space.

- blow-spherical equivalence (or blow-spherical homeomorphism) (between $\left(X, x_{0}\right)$ and $\left.\left(Y, y_{0}\right)\right)$, if the mapping

$$
\rho_{m, y_{0}}^{-1} \circ \varphi \circ \rho_{n, x_{0}}: X_{x_{0}}^{\prime} \backslash \partial X_{x_{0}}^{\prime} \rightarrow Y_{y_{0}}^{\prime} \backslash \partial Y_{y_{0}}^{\prime}
$$

extends as a homeomorphism $\varphi^{\prime}: X_{x_{0}}^{\prime} \rightarrow Y_{y_{0}}^{\prime}$. In this case, we say that $\left(X, x_{0}\right)$ and $\left(Y, y_{0}\right)$ are blow-spherical equivalent. We denote by $H_{B S}$ to be the set of all blow-spherical equivalences between germs of complex analytic sets at the origin of some complex Euclidean space.

- bi-Lipschitz equivalence (between $\left(X, x_{0}\right)$ and $\left(Y, y_{0}\right)$ ), if $h$ is a biLipschitz mapping with respect the outer metrics of $\mathbb{R}^{n}$ and $\mathbb{R}^{m}$. In this case, we say that $\left(X, x_{0}\right)$ and $\left(Y, y_{0}\right)$ are bi-Lipschitz equivalent. We denote by $H_{L i p}$ to be the set of all bi-Lipschitz equivalences between germs of complex analytic sets at the origin of some complex Euclidean space.

Proposition 2.7. The sets $H_{D i f f\left(C^{1}\right)}, H_{D i f f}, H_{w D i r}, H_{B S}$ and $H_{\text {Lip }}$ are classes of germs of homeomorphisms that preserve tangent cones.

Proof. It is easy to see that $H_{D i f f\left(C^{1}\right)} \subset H_{\text {Diff }}$. Moreover, if $h \in H_{\text {Diff }}$, then it is also easy to see that $D h_{0}$ and $D h_{0}^{-1}$ are restrictions of linear mappings and $D h_{0}: C(X, 0) \rightarrow C(Y, 0)$ is a homeomorphism and, in particular, $D h_{0} \in H_{D i f f\left(C^{1}\right)}$. 
This tell us that $H_{D i f f\left(C^{1}\right)}$ and $H_{D i f f}$ are classes of germs of homeomorphisms that preserve tangent cones. About the classes $H_{B S}$ and $H_{L i p}$ this was shown, respectively, in (14, Proposition 3.2) and in ([13, Theorem 3.2).

Hence, if $h \in H_{w D i r}$, then $D^{+} h_{0}: C(X, 0) \rightarrow C(Y, 0)$ is a homeomorphism. To finish the proof it is enough to show that $D^{+} h_{0} \in H_{w D i r}$. However, $D^{+} h_{0}$ : $C(X, 0) \rightarrow C(Y, 0)$ is a positive homogeneous mapping (i.e., $D^{+} h_{0}(\lambda v)=\lambda D^{+} \varphi_{0}(v)$ for all $\lambda \geq 0$ and $v \in C(X, 0))$, then for $v \in C(X, 0)$ and $\alpha:[0, \varepsilon) \rightarrow C(X, 0)$ being a curve satisfying $\alpha(0)=0$ and $\alpha(t)=t v+o(t)$, we obtain

$$
\lim _{t \rightarrow 0^{+}} \frac{D^{+} h_{0}(\alpha(t))}{t}=\lim _{t \rightarrow 0^{+}} D^{+} h_{0}\left(\frac{\alpha(t)}{t}\right)=D^{+} h_{0}(v)
$$

since $D^{+} h_{0}$ is a continuous mapping and $\lim _{t \rightarrow 0^{+}} \frac{\alpha(t)}{t}=v$. Therefore, $D^{+} h_{0}$ is weakly directional differentiable with $D^{+}\left(D^{+} h_{0}\right)_{0}=D^{+} h_{0}$. By the same way, $D^{+} h_{0}^{-1}$ is weakly directional differentiable as well and as $D^{+}\left(D^{+} h_{0}\right)_{0}=D^{+} h_{0}$ is a homeomorphism, we obtain that $D^{+} h_{0} \in H_{w D i r}$.

Definition 2.8. We say that a homeomorphism $\varphi:\left(\mathbb{C}^{n}, 0\right) \rightarrow\left(\mathbb{C}^{n}, 0\right)$ satisfies Question B' (resp. Question B") if for any complex analytic functions $f, g$ : $\left(\mathbb{C}^{n}, 0\right) \rightarrow(\mathbb{C}, 0)$ such that $\varphi(V(f))=V(g)$, we have that there exists a homeomorphism $\phi: \mathbb{P} C(V(f), 0) \rightarrow \mathbb{P} C(V(g), 0)$ (resp. $\phi:(C(X, 0), 0) \rightarrow(C(Y, 0), 0))$.

Corollary 2.9. All element of $H_{D i f f\left(C^{1}\right)}, H_{D i f f}, H_{w D i r}, H_{B S}$ and $H_{\text {Lip }}$ satisfies Question B".

Proposition 2.10. Let $(X, 0)$ and $(Y, 0)$ be germs of subanalytic subsets. If $\varphi:(X, 0) \rightarrow(Y, 0)$ is a weak directional equivalence, then $\varphi$ is a blow-spherical homeomorphism.

Proof. As $\varphi:(X, 0) \rightarrow(Y, 0)$ is a weak directional equivalence, then $D^{+} \varphi_{0}$ : $C(X, 0) \rightarrow C(Y, 0)$ is a homeomorphism. In particular, the mapping $\nu_{\varphi}: \mathbb{S}_{0} X \rightarrow$ $\mathbb{S}_{0} Y$ given by $\nu_{\varphi}(v)=\frac{D^{+} \varphi_{0}(v)}{\left\|D^{+} \varphi_{0}(v)\right\|}$ is a homeomorphism, since the mapping $D^{+} \varphi_{0}$ : $C(X, 0) \rightarrow C(Y, 0)$ is a positive homogeneous mapping (i.e., $D^{+} \varphi_{0}(\lambda v)=\lambda D^{+} \varphi_{0}(v)$ for all $\lambda \geq 0$ and $v \in C(X, 0))$.

Therefore, the mapping $\varphi^{\prime}: X^{\prime} \rightarrow Y^{\prime}$ given by

$$
\varphi^{\prime}(x, t)= \begin{cases}\left(\frac{\varphi(t x)}{\|\varphi(t x)\|},\|\varphi(t x)\|\right), & \text { if } t \neq 0 \\ \left(\nu_{\varphi}(x), 0\right), & \text { if } t=0\end{cases}
$$

is a homeomorphism.

Corollary 2.11. $H_{\text {Diff }\left(C^{1}\right)} \subset H_{\text {Diff }} \subset H_{w \text { Dir }} \subset H_{B S}$.

\section{Invariance of the multiplicity and counter examples}

In [8], it was shown that the multiplicity of complex analytic set is invariant by a differentiable equivalence. Thus, the classes $H_{D i f f\left(C^{1}\right)}$ and $H_{D i f f}$ preserve the multiplicity. Additionally, in [2 it was shown that the multiplicity of a complex analytic curve or surface is invariant by a bi-Lipschitz homeomorphism and in $\mathbf{3}$ ] it was shown that in general the multiplicity of complex analytic set with dimension greater than 2 is not invariant by a bi-Lipschitz homeomorphism. In this Section we study the invariance of the multiplicity with respect the classes $H_{w D i r}$ and $H_{B S}$. 
Theorem 3.1. Let $X \subset \mathbb{C}^{n}$ and $Y \subset \mathbb{C}^{m}$ be two complex analytic sets with the same dimension. Suppose that $1 \leq \operatorname{dim} X \leq 2$. If $(X, 0)$ and $(Y, 0)$ are blowspherical homeomorphic, then $m(X, 0)=m(Y, 0)$.

Proof. By Theorem 5.1 in [14, it is enough to show Theorem 3.1 when $X$ and $Y$ are two irreducible homogeneous complex algebraic sets. The Theorem 5.1 in 14 was stated with the sets $X$ and $Y$ being subsets in same ambient space, however, let me emphasize that the same proof of the Theorem 5.1 in [14] works when the sets $X$ and $Y$ are subsets in different ambient spaces. Thus, we suppose that $X$ and $Y$ are two irreducible homogeneous complex algebraic sets and they are blow-spherical homeomorphic. Therefore, if $\operatorname{dim} X=\operatorname{dim} Y=1$, then by Theorem 6.1 in 14, we have $m(X, 0)=m(Y, 0)$. Thus, we can suppose that $\operatorname{dim} X=$ $\operatorname{dim} Y=2$. However, since $(X, 0)$ and $(Y, 0)$ are blow-spherical homeomorphic, then they are, in particular, homeomorphic and, therefore, by Proposition 3.5 in [2], we obtain $m(X, 0)=m(Y, 0)$, since degree and multiplicity are equal for homogeneous complex algebraic sets.

Proposition 2.10 jointly with Theorem 3.1 give us the following result.

Corollary 3.2. Let $X \subset \mathbb{C}^{n}$ and $Y \subset \mathbb{C}^{m}$ be two complex analytic sets with the same dimension. Suppose that $1 \leq \operatorname{dim} X \leq 2$. If $(X, 0)$ and $(Y, 0)$ are weak directional equivalent, then $m(X, 0)=m(Y, 0)$.

However, in the other dimensions the above results are not true, as it is shown in the next theorem.

TheOREM 3.3. For each $n \geq 3$, there exists a family $\left\{Y_{i}\right\}_{i \in \mathbb{Z}}$ of $n$-dimensional complex algebraic varieties $Y_{i} \subset \mathbb{C}^{n_{i}+1}$ such that:

(a) for each pair $i \neq j$, the germs at the origin of $Y_{i} \subset \mathbb{C}^{n_{i}+1}$ and $Y_{j} \subset$ $\mathbb{C}^{n_{j}+1}$ are weak directional equivalent, but $\left(Y_{i}, 0\right)$ and $\left(Y_{j}, 0\right)$ have different multiplicity.

(b) for each pair $i \neq j$, the germs at the origin of $Y_{i} \subset \mathbb{C}^{n_{i}+1}$ and $Y_{j} \subset$ $\mathbb{C}^{n_{j}+1}$ are blow-spherical equivalent, but $\left(Y_{i}, 0\right)$ and $\left(Y_{j}, 0\right)$ have different multiplicity.

Proof. Let $\left\{p_{i}\right\}_{i \in \mathbb{Z}}$ be the family of odd prime numbers. In the proof of Theorem in $\left[3\right.$ it was shown that there exists a family $\left\{X_{i}\right\}_{i \in \mathbb{Z}}$ of 2-dimensional projective varieties, such that each $X_{i} \subset \mathbb{C} P^{m_{i}}$ is obtained by the embedding of a very ample bundle $L_{i}$, where $L_{i}$ is a very ample bundle on $X=\mathbb{C} P^{1} \times \mathbb{C} P^{1}$ of bidegree $\left(2, p_{i}\right)$. For each $i \in \mathbb{Z}$ we denote by $S^{2 m_{i}+1}$ to be the unit sphere centered in $0 \in \mathbb{C}^{m_{i}+1}$ and by $C\left(X_{i}\right) \subset \mathbb{C}^{m_{i}+1}$ to be the affine cone of the projective variety $X_{i}$. Thus, it is also shown in [3] that for each pair $i \neq j$ the links $S_{i}:=$ $C\left(X_{i}\right) \cap S^{2 m_{i}+1}$ and $S_{j}:=C\left(X_{j}\right) \cap S^{2 m_{j}+1}$ are diffeomorphic to $S^{2} \times S^{3}$ and, in particular, $S_{i}$ and $S_{j}$ are bi-Lipschitz homeomorphic. Moreover, $m\left(C\left(X_{i}\right), 0\right)=4 p_{i}$, for all $i \in \mathbb{Z}$. Let $\phi: S_{i} \rightarrow S_{j}$ be a bi-Lipschitz homeomorphism. Then, the mapping $\Phi: C\left(X_{i}\right) \rightarrow C\left(X_{j}\right)$ given by

$$
\Phi(x)= \begin{cases}\|x\| \cdot \phi\left(\frac{x}{\|x\|}\right), & \text { if } x \neq 0 \\ 0, & \text { if } x=0\end{cases}
$$

is a bi-Lipschitz homeomorphism as well. Thus, for each $k \in \mathbb{Z}$ we define $Y_{k}:=$ $C\left(X_{k}\right) \times \mathbb{C}^{n-3}$. Then the mapping $\Psi: Y_{i} \rightarrow Y_{j}$ given by $\Psi(x, y)=(\Phi(x), y)$ for all $(x, y) \in C\left(X_{i}\right) \times \mathbb{C}^{n-3}$ is also a bi-Lipschitz homeomorphism. 
Claim. $\Psi:\left(Y_{i}, 0\right) \rightarrow\left(Y_{j}, 0\right)$ is a weak directional equivalence.

Firstly, we remark that if $\Psi$ is weak directional differentiable, then $D^{+} \Psi_{0}=\Psi$. In fact, since $\Psi$ is a positive homogeneous mapping and $Y_{i}$ is a cone, we have

$$
D^{+} \Psi_{0}(v)=\lim _{t \rightarrow 0^{+}} \frac{\Psi(t v)}{t}=\lim _{t \rightarrow 0^{+}} \frac{t \Psi(v)}{t}=\Psi(v),
$$

for all $v \in Y_{i}$. The same statement is true for $\Psi^{-1}$, since $\Psi^{-1}$ is also a positive homogeneous mapping and $Y_{j}$ is a cone as well. Thus, let $v$ be a point in $C\left(Y_{i}, 0\right)=$ $Y_{i}$ and $\alpha:[0, \varepsilon) \rightarrow Y_{i}$ be a curve satisfying $\alpha(0)=0$ and $\alpha(t)=t v+o(t)$. Using the fact that $\Psi$ is Lipschitz, we obtain that $\Psi(\alpha(t))-\Psi(t v)=o(t)$. Then,

$$
\lim _{t \rightarrow 0^{+}} \frac{\Psi(\alpha(t))}{t}=\lim _{t \rightarrow 0^{+}} \frac{\Psi(t v)}{t}=\Psi(v) .
$$

Thus, $\Psi$ is weak directional differentiable and by the same way $\Psi^{-1}$ is also weak directional differentiable. As $D^{+} \Psi_{0}=\Psi$ is a homeomorphism, we have that $\Psi$ is a weak directional equivalence.

Then, for each pair $i \neq j,\left(Y_{i}, 0\right)$ and $\left(Y_{j}, 0\right)$ are weak directional equivalent. Therefore, we have that the family $\left\{Y_{i}\right\}_{i \in \mathbb{Z}}$ satisfies the item (a), since $m\left(Y_{i}, 0\right)=$ $m\left(C\left(X_{i}\right), 0\right)=4 p_{i}$, for all $i \in \mathbb{Z}$.

In order to obtain the item (b) and finish the proof we just need to recall Proposition 2.10.

\section{Invariance of the multiplicity by right equivalence}

Let me introduce a new class of homeomorphisms that essentially generalizes biLipschitz equivalence, weak directional equivalence, differentiable equivalence and $C^{1}$ equivalence.

Definition 4.1. For each $n \in \mathbb{N}$, let $\mathcal{H}_{n}$ be the collection of all germs of homeomorphisms from $\left(\mathbb{C}^{n}, 0\right)$ to $\left(\mathbb{C}^{n}, 0\right)$. Let $\mathcal{T}_{\mathcal{H}} \subset \mathcal{H}:=\bigcup_{n \in \mathbb{N}} \mathcal{H}_{n}$ (resp. $\mathcal{T}_{\mathbb{P} \mathcal{H}} \subset$ $\left.\mathcal{H}:=\bigcup_{n \in \mathbb{N}} \mathcal{H}_{n}\right)$ be the maximal subset with respect the inclusion satisfying:

i) If $\varphi \in \mathcal{T}_{\mathcal{H}}\left(\right.$ resp. $\left.\varphi \in \mathcal{T}_{\mathbb{P} \mathcal{H}}\right)$, then $\varphi \times \mathrm{id}_{2} \in \mathcal{T}_{\mathcal{H}}\left(\right.$ resp. $\left.\varphi \times \mathrm{id}_{2} \in \mathcal{T}_{\mathbb{P} \mathcal{H}}\right)$, where $\operatorname{id}_{2}:(\mathbb{C}, 0) \rightarrow(\mathbb{C}, 0)$ is the germ of the identity mapping;

ii) If $\varphi \in \mathcal{T}_{\mathcal{H}}\left(\right.$ resp. $\left.\varphi \in \mathcal{T}_{\mathbb{P} \mathcal{H}}\right)$, then $\varphi^{-1} \in \mathcal{T}_{\mathcal{H}}\left(\right.$ resp. $\left.\varphi^{-1} \in \mathcal{T}_{\mathbb{P} \mathcal{H}}\right)$;

iii) Any $\varphi \in \mathcal{T}_{\mathcal{H}}\left(\right.$ resp. $\left.\varphi \in \mathcal{T}_{\mathbb{P} \mathcal{H}}\right)$ satisfies Question B" (resp. Question B').

Remark 4.2. It is easy to verify that each set $H_{D i f f\left(C^{1}\right)}, H_{D i f f}, H_{w-D i r}$ and $H_{\text {Lip }}$ intersected with $\mathcal{H}$ is a subset of $\mathcal{T}_{\mathcal{H}}$.

TheOREM 4.3. Let $f, g:\left(\mathbb{C}^{n}, 0\right) \rightarrow(\mathbb{C}, 0)$ be complex analytic functions.

(a) If there exists $\varphi \in \mathcal{T}_{\mathcal{H}}$ such that $f=g \circ \varphi$, then $\operatorname{ord}_{0} f=\operatorname{ord}_{0} g$.

(b) If there exists $\varphi \in \mathcal{T}_{\mathbb{P} \mathcal{H}}$ such that $f=g \circ \varphi$, then $\operatorname{ord}_{0} f=\operatorname{ord}_{0} g$.

Proof. Suppose firstly that $\varphi \in \mathcal{H}_{n}$ and $f=g \circ \varphi$. Suppose that $\operatorname{ord}_{0} f<$ $\operatorname{ord}_{0} g$. If $k=\operatorname{ord}_{0} f$, then by Lê-A'Campo's Theorem (see [1] and [10]), we have that $k>1$. Let us define $\tilde{f}, \tilde{g}:\left(\mathbb{C}^{n} \times \mathbb{C}, 0\right) \rightarrow(\mathbb{C}, 0)$ by $\tilde{f}(x, t)=f(x)+t^{k}$ and $\tilde{g}(x, t)=g(x)+t^{k}$. Then, $C(V(\tilde{g}), 0)=\{t=0\}=\mathbb{C}^{n} \times\{0\}$. Moreover, since $f_{k}:=\operatorname{in} f \not \equiv 0$ and $k>1$, we get that $f_{k}+t^{k}$ cannot be a power of a linear form and, in particular, $C(V(\tilde{f}), 0)=V\left(f_{k}+t^{k}\right)$ is not a linear subspace. Now, let us define $\tilde{\varphi}:\left(\mathbb{C}^{n} \times \mathbb{C}, 0\right) \rightarrow\left(\mathbb{C}^{n} \times \mathbb{C}, 0\right)$ by $\tilde{\varphi}(x, t)=(\varphi(x), t)$. Therefore, $\tilde{f}=\tilde{g} \circ \tilde{\varphi}$. 
Proof of item (a). If $\varphi \in \mathcal{T}_{\mathcal{H}}$, by item i) of the definition 4.1 we have that $\tilde{\varphi} \in \mathcal{T}_{\mathcal{H}}$. By item iii) of the definition 4.1. there exists a homeomorphism $\tilde{\phi}:\left(V\left(f_{k}+t^{k}\right), 0\right) \rightarrow$ $\left(\mathbb{C}^{n} \times\{0\}, 0\right)$. Thus, by Prill's Theorem (Theorem in [1] $), V\left(f_{k}+t^{k}\right)$ is a linear subspace of $\mathbb{C}^{n}$, which is a contradiction. Then, $\operatorname{ord}_{0} f \leq \operatorname{ord}_{0} g$. Since by item ii) of the definition 4.1. $\varphi^{-1} \in \mathcal{T}_{\mathcal{H}}$, we obtain also that $\operatorname{ord}_{0} g \leq \operatorname{ord}_{0} f$. Therefore, $\operatorname{ord}_{0} f=\operatorname{ord}_{0} g$.

Proof of item (b). If $\varphi \in \mathcal{T}_{\mathbb{P} \mathcal{H}}$, by item i) of the definition 4.1. we have that $\tilde{\varphi} \in \mathcal{T}_{\mathbb{P} \mathcal{H}}$. By item iii) of the definition 4.1, there exists a homeomorphism $\tilde{\phi}: \mathbb{P} V\left(f_{k}+t^{k}\right) \rightarrow$ $\mathbb{P}\left(\mathbb{C}^{n} \times\{0\}\right)$. If $\operatorname{dim} \mathbb{P} C(V(\tilde{f}), 0) \neq 2$, by Corollary 2.12 in $([\mathbf{5}$, p. 145), $\mathbb{P} C(V(\tilde{f}), 0)$ is a linear subspace of $\mathbb{C} P^{n}$, since $\mathbb{P}\left(\mathbb{C}^{n} \times\{0\}\right) \cong \mathbb{C} P^{n-1}$. In particular, $C(V(\tilde{f}), 0)$ is a linear subspace of $\mathbb{C}^{n+1}$, which is a contradiction. If $\operatorname{dim} \mathbb{P} C(V(\tilde{f}), 0)=2$ and, in particular, $n=3$, then we define $\bar{f}, \bar{g}:\left(\mathbb{C} \times \mathbb{C}^{3} \times \mathbb{C}, 0\right) \rightarrow(\mathbb{C}, 0)$ by $\bar{f}(s, x, t)=\tilde{f}(x, t)+s^{k+1}$ and $\bar{g}(s, x, t)=\tilde{g}(x, t)+s^{k+1}$ and we define $\bar{\varphi}:(\mathbb{C} \times$ $\left.\mathbb{C}^{3} \times \mathbb{C}, 0\right) \rightarrow\left(\mathbb{C} \times \mathbb{C}^{3} \times \mathbb{C}, 0\right)$ by $\bar{\varphi}(s, x, t)=(s, \varphi(x), t)$. Therefore, $\bar{f}=\bar{g} \circ \bar{\varphi}$. Moreover, $C(V(\bar{g}), 0)=\{t=0\}=\mathbb{C}^{4} \times\{0\} \subset \mathbb{C}^{5}, C(V(\bar{f}), 0)=V\left(f_{k}+t^{k}\right) \subset \mathbb{C}^{5}$ and as before $C(V(\bar{f}), 0)$ cannot be a linear subspace. However, since $\tilde{\varphi} \in \mathcal{T}_{\mathbb{P} \mathcal{H}}$, by item i) of the definition 4.1 we have that $\bar{\varphi} \in \mathcal{T}_{\mathbb{P} \mathcal{H}}$. Then, By item iii) of the definition 4.1. there exists a homeomorphism $\bar{\phi}: \mathbb{P} C(V(\bar{f}), 0) \rightarrow \mathbb{P}\left(\mathbb{C}^{n+1} \times\{0\}\right)$. So, since $\operatorname{dim} \mathbb{P} C(V(\bar{f}), 0)=3 \neq 2$, by the same reason as before, $C(V(\bar{f}), 0)$ is a linear subspace of $\mathbb{C}^{n+2}$, which is a contradiction.

Then, in any case, $\operatorname{ord}_{0} f \leq \operatorname{ord}_{0} g$. Since by item ii) of the definition 4.1. $\varphi^{-1} \in \mathcal{T}_{\mathbb{P} \mathcal{H}}$, we obtain also that $\operatorname{ord}_{0} g \leq \operatorname{ord}_{0} f$. Therefore, $\operatorname{ord}_{0} f=\operatorname{ord}_{0} g$.

REMARK 4.4. It is clear of the proof of Theorem 4.3 that if Question B' (or Question B") has a positive answer when we consider only right equivalence, then Question A' has a positive answer as well.

Definition 4.5. Let $F=\left(f_{1}, \cdots, f_{m}\right):\left(\mathbb{C}^{n}, 0\right) \rightarrow \mathbb{C}^{m}$ be a complex analytic mapping. We define the order of $F$ at $0 \in \mathbb{C}^{n}$ by

$$
\operatorname{ord}_{0}(F)=\max \left\{\operatorname{ord}_{0}\left(f_{1}\right), \cdots, \operatorname{ord}_{0}\left(f_{m}\right)\right\} .
$$

Corollary 4.6. Let $F, G: \mathbb{C}^{n} \rightarrow \mathbb{C}^{m}$ be complex analytic mappings. If there exists $\varphi \in \mathcal{T}_{\mathcal{H}} \cup \mathcal{T}_{\mathbb{P} \mathcal{H}}$ such that $F=G \circ \varphi$, then $\operatorname{ord}(F)=\operatorname{ord}(G)$.

\section{Invariance of the degree by right equivalence}

Definition 5.1. Let $A \subset \mathbb{R}^{n}$ be an unbounded subset. We say that $v \in \mathbb{R}^{n}$ is a tangent vector of $A$ at infinity if there exist a continuous curve $\alpha:(\varepsilon, \infty) \rightarrow A$ satisfying $\alpha(t)=t v+o_{\infty}(t)$, where $g(t)=o_{\infty}(t)$ means that $\lim _{t \rightarrow+\infty} \frac{g(t)}{t}=0$. Let $C_{\infty}(A)$ denote the set of all tangent vectors of $A$ at infinity. This subset $C_{\infty}(A) \subset$ $\mathbb{R}^{n}$ is called the tangent cone of $A$ at infinity.

Definition 5.2. For each $n \in \mathbb{N}$, let $\mathcal{H}_{n, \infty}$ be the collection of all germs of homeomorphisms at infinity from $\mathbb{C}^{n}$ to $\mathbb{C}^{n}$. Let $\mathcal{T}_{\mathcal{H}, \infty} \subset \mathcal{H}_{\infty}:=\bigcup_{n \in \mathbb{N}} \mathcal{H}_{n, \infty}$ (resp. $\left.\mathcal{T}_{\mathbb{P} \mathcal{H}, \infty} \subset \mathcal{H}_{\infty}\right)$ be the maximal subset with respect the inclusion satisfying:

i) If $\varphi \in \mathcal{T}_{\mathcal{H}, \infty}\left(\right.$ resp. $\left.\varphi \in \mathcal{T}_{\mathbb{P} \mathcal{H}, \infty}\right)$, then $\varphi \times \operatorname{id}_{2} \in \mathcal{T}_{\mathcal{H}, \infty}\left(\right.$ resp. $\varphi \times \operatorname{id}_{2} \in$ $\left.\mathcal{T}_{\mathbb{P} \mathcal{H}, \infty}\right)$, where $\operatorname{id}_{2}:(\mathbb{C}, 0) \rightarrow(\mathbb{C}, 0)$ is the germ of the identity mapping;

ii) If $\varphi \in \mathcal{T}_{\mathcal{H}, \infty}\left(\right.$ resp. $\left.\varphi \in \mathcal{T}_{\mathbb{P} \mathcal{H}, \infty}\right)$, then $\varphi^{-1} \in \mathcal{T}_{\mathcal{H}, \infty}\left(\operatorname{resp} . \varphi^{-1} \in \mathcal{T}_{\mathbb{P} \mathcal{H}, \infty}\right)$; 
iii) If $\varphi \in \mathcal{T}_{\mathcal{H}, \infty}\left(\right.$ resp. $\left.\varphi \in \mathcal{T}_{\mathbb{P} \mathcal{H}, \infty}\right)$ and $f, g: \mathbb{C}^{n} \rightarrow \mathbb{C}$ are complex polynomials such that $\varphi(V(f))=V(g)$ (outside of a compact subset), then there exists a homeomorphism $\phi:\left(C_{\infty}(X), 0\right) \rightarrow\left(C_{\infty}(Y), 0\right)$ (resp. $\phi: \mathbb{P} C_{\infty}(X) \rightarrow$ $\left.\mathbb{P} C_{\infty}(Y)\right)$.

EXAMPLE 5.3. If $\operatorname{Lip}_{n, \infty}$ is the collection of all germs of bi-Lipschitz homeomorphisms at infinity from $\mathbb{C}^{n}$ to $\mathbb{C}^{n}$, then by Theorem 4.5 in $[7$, it is easy to verify that $\operatorname{Lip}_{n, \infty} \subset \mathcal{T}_{\mathcal{H}, \infty}$.

As an application of the proof of Theorem 4.3 , we obtain a result about invariance of the degree.

Corollary 5.4. Let $f, g: \mathbb{C}^{n} \rightarrow \mathbb{C}$ be complex polynomials. If there exists $\varphi \in \mathcal{T}_{\mathcal{H}, \infty}$ such that $f=g \circ \varphi$ (outside of a compact subset), then $\operatorname{deg}(f)=\operatorname{deg}(g)$.

Proof. Suppose that $\operatorname{deg}(f)>\operatorname{deg}(g)$. If $k=\operatorname{deg}(f)$, then we have that $k>1$, since $g$ cannot be constant. Let $f_{k}$ be the homogeneous polynomial formed by the monomials of $f$ that have degree $k$. Let us define $\tilde{f}, \tilde{g}:\left(\mathbb{C}^{n+1}, 0\right) \rightarrow(\mathbb{C}, 0)$ by $\tilde{f}(x, t)=f(x)+t^{k}$ and $\tilde{g}(x, t)=g(x)+t^{k}$. Then, $C(V(\tilde{g}), 0)=\{t=0\}=\mathbb{C}^{n} \times\{0\}$. Moreover, since $f_{k} \not \equiv 0$ and $k>1$, we get that $f_{k}+t^{k}$ cannot be a power of a linear form and, in particular, $C(V(\tilde{f}), 0)=V\left(f_{k}+t^{k}\right)$ is not a linear subspace. Now, let us define $\tilde{\varphi}:\left(\mathbb{C}^{n+1}, 0\right) \rightarrow\left(\mathbb{C}^{n+1}, 0\right)$ by $\tilde{\varphi}(x, t)=(\varphi(x), t)$. By item i) of the definition 5.2 , we have that $\tilde{\varphi} \in \mathcal{T}_{\mathcal{H}, \infty}$. By item iii) of the definition 5.2 , there exists a homeomorphism $\tilde{\phi}:\left(V\left(f_{k}+t^{k}\right), 0\right) \rightarrow\left(\mathbb{C}^{n} \times\{0\}, 0\right)$. Thus, by Prill's Theorem (Theorem in [1]), $V\left(f_{k}+t^{k}\right)$ is a linear subspace, which is a contradiction.

REMARK 5.5. We can also prove a result like Corollary 5.4, when $\varphi \in \mathcal{T}_{\mathbb{P} \mathcal{H}, \infty}$.

We would like to say that in general it is hard that the degree to be preserved by some equivalence, as we can see in next example.

ExAmple 5.6. Let $f, g: \mathbb{C}^{n} \rightarrow \mathbb{C}$ be two complex polynomials such that $f(x, y)=y-x^{2}$ and $g(x, y)=y$. Let $\varphi: \mathbb{C}^{2} \rightarrow \mathbb{C}^{2}$ be the polynomial diffeomorphism given by $\varphi(x, y)=\left(x, y-x^{2}\right)$. Then $f=g \circ \varphi$. However, $\operatorname{deg}(f)=2$ and $\operatorname{deg}(g)=1$.

In particular, a polynomial diffeomorphism does not need to belong to $\mathcal{T}_{\mathcal{H}, \infty}$.

Definition 5.7. Let $F=\left(f_{1}, \cdots, f_{m}\right): \mathbb{C}^{n} \rightarrow \mathbb{C}^{m}$ be a polynomial mapping. We define the degree of $F$ by

$$
\operatorname{deg}(F)=\max \left\{\operatorname{deg}\left(f_{1}\right), \cdots, \operatorname{deg}\left(f_{m}\right)\right\} .
$$

Corollary 5.8. Let $F, G: \mathbb{C}^{n} \rightarrow \mathbb{C}^{k}$ be complex polynomials mappings. If there exists $\varphi \in \mathcal{T}_{\mathcal{H}, \infty} \cup \mathcal{T}_{\mathbb{P} \mathcal{H}, \infty}$ such that $F=G \circ \varphi$ (outside of a compact subset), then $\operatorname{deg}(F)=\operatorname{deg}(G)$.

\section{References}

[1] A'campo, N. Le nombre de Lefschetz d'une monodromie. (French) Nederl. Akad. Wetensch. Proc. Ser. A 76 = Indag. Math., vol. 35 (1973), 113-118.

[2] Fernandez de Bobadilla, J.; Fernandes, A. and Sampaio, J. Edson Multiplicity and degree as bi-Lipschitz invariants for complex sets. arXiv:1706.06614v2 [math.AG], 2017.

[3] Birbrair, L.; Fernandes, A.; Sampaio, J. Edson and Verbitsky, M. Multiplicity of singularities is not a bi-Lipschitz invariant. arXiv:1801.06849v1 [math.AG], 2018.

[4] J. F. Bobadilla. Answers to some equisingularity questions. Invent. math., vol. 161 (2005), 657-675. 
[5] Dimca, A. Singularities and Topology of Hypersurfaces. Springer-Verlag, New York, 1992.

[6] Fernandes, A. and Sampaio, J. Edson. Multiplicity of analytic hypersurface singularities under bi-Lipschitz homeomorphisms. Journal of Topology, vol. 9 (2016), 927-933.

[7] Fernandes, A. and Sampaio, J. Edson. On Lipschitz rigidity of complex analytic sets. arXiv:1705.03085v3 [math.AG], 2018.

[8] Gau, Y.-N. and Lipman, J. Differential invariance of multiplicity on analytic varieties. Inventiones mathematicae, vol. 73 (1983), no. 2, 165-188.

[9] King, Henry C. Topological Type of Isolated Critical Points. Annals of Mathematics, Second Series, vol. 107 (1978), no. 3, 385-397.

[10] Lê D. T. Calcul du nombre de cycles évanouissants d'une hypersurface complexe. (French) Ann. Inst. Fourier (Grenoble), vol. 23 (1973), no. 4, 261-270.

[11] Prill, D. Cones in complex affine space are topologically singular. Proc. of AMS, vol. 18 (1967), 178-182.

[12] Saeki, O. Topological types of complex isolated hypersurface singularities. Kodai Math. J., vol. 12 (1989), 23-29.

[13] Sampaio, J. Edson Bi-Lipschitz homeomorphic subanalytic sets have bi-Lipschitz homeomorphic tangent cones. Selecta Mathematica: New Series, vol. 22 (2016), no. 2, 553-559.

[14] Sampaio, J. Edson Multiplicity, regularity and blow-spherical equivalence of complex analytic sets. arXiv:1702.06213v2 [math.AG], 2017.

[15] Shapiro, A. On Concepts of Directional Differentiability. Journal of optimization theory and applications, vol. 66 (1990), no. 3, 477-487.

[16] Zariski, O. Some open questions in the theory of singularities. Bull. of the Amer. Math. Soc., vol. 77 (1971), no. 4, 481-491.

(J. Edson Sampaio) (1) Departamento de Matemática, Universidade Federal do Ceará, Rua Campus do Pici, s/N, Bloco 914, Pici, 60440-900, Fortaleza-CE, Brazil. E-mail: edsonsampaio@mat.ufc.br

(2) BCAM - Basque Center for Applied Mathematics, Mazarredo, 14 E48009 Bilbao, BAsque Country - Spain. E-mail: esampaio@bcamath.org 